\title{
Single-step QuantiFERON screening of adult contacts: a prospective cohort study of tuberculosis risk
}

\author{
Pranabashis Haldar, ${ }^{1,2}$ Helen Thuraisingam, ${ }^{1}$ Hemu Patel, ${ }^{3}$ Nelun Pereira, ${ }^{3}$ \\ Robert C Free, ${ }^{2}$ James Entwisle, ${ }^{1}$ Martin Wiselka, ${ }^{4}$ Edwyn Wren Hoskyns, ${ }^{5}$

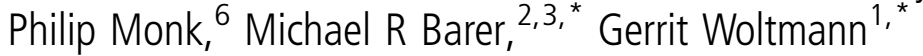

\begin{abstract}
- Additional supplementary figures and tables are published online only. To view this file please visit the journal online (http://dx.doi.org/10. 1136/thoraxjnl-2011-200956).

${ }^{1}$ Department of Respiratory Medicine, Institute for Lung Health, Glenfield Hospital, Leicester, UK

${ }^{2}$ Department of Infection, Immunity and Inflammation, University of Leicester, Leicester, UK

${ }^{3}$ Department of Microbiology, Leicester Royal Infirmary, Leicester, UK

${ }^{4}$ Department of Infectious Diseases, Leicester Royal Infirmary, Leicester, UK ${ }^{5}$ Department of Paediatrics and Neonatal Medicine, Leicester Royal Infirmary, Leicester, UK ${ }^{6}$ Health Protection Agency, East Midlands South Health Protection Unit, Leicester, UK *Cosenior authorship for manuscript.
\end{abstract}

\section{Correspondence to} Dr Pranab Haldar, Department of Respiratory Medicine, Glenfield Hospital, Groby Road Leicester, LE3 9QP, UK; ph62@le.ac.uk

Received 16 August 2011 Revised 6 August 2012 Accepted 9 August 2012 Published Online First 6 September 2012

\section{SLinked}

http://dx.doi.org/10.1136/ thoraxjnl-2011-201542

To cite: Haldar $\mathrm{P}$, Thuraisingam $\mathrm{H}$, Patel $\mathrm{H}$, et al. Thorax 2013;68: 240-246.

\section{ABSTRACT}

Background The efffectiveness of tuberculosis (TB) contact screening programmes using interferon $\gamma$ release assays remains uncertain as prospective contact TB risk is not well characterised.

Objectives To quantify 2-year TB risk and evaluate screening performance with single-step QuantiFERON TB Gold-In Tube (QFT) in adult contacts. To compare TB risk between QFT tested subgroups stratified by exposure type (smear positive pulmonary (SP) versus non-smear positive (NSP) TB) and age (younger ( $16-35$ years) versus older ( $\geq 36$ years)).

Methods Screening involved QFT testing in older contacts of SP and all younger contacts, 8-12 weeks after index notification. Chemoprevention (3RH) was offered to QFT positive (+) younger adults. TB risk was determined in a prospective cohort study.

Results 43 TB events occurred in 1769 adult contacts observed for median 717 days (2-year rate $(95 \% \mathrm{Cl})$ $=2.5 \%$ (1.7 to 3.2)). Index-contact strain matching was demonstrable for 18 of 22 (82\%) paired samples. No contacts (0/98) receiving 3RH developed TB. 215 of 817 appropriately tested adults (26.3\%) were QFT+. 14 of 112 untreated QFT+ adults developed TB (2-year rate $(95 \% \mathrm{Cl})=13.4 \%$ (7.7 to 21.1$))$. The model required 35 contacts screened with QFT to identify one contact developing TB at 2 years. TB rates were comparable in $\mathrm{QFT}+$ contacts of SP and NSP (rate ratio $(\mathrm{RR})=0.98$, $\mathrm{p}=0.962)$. For QFT+ older contacts, the disease rate was lower $(8.9 \%$ (3.3 to 19.1)) and similar to the overall group rate $(R R=1.4, p=0.503)$.

Conclusions QFT based single-step contact screening is effective in young adults.

\section{INTRODUCTION}

Preventing future tuberculosis (TB) requires screening programmes to identify and treat latent infection with Mycobacterium tuberculosis (LTBI) in high risk populations. However, LTBI is associated with a variable future risk of active TB that is complex, incompletely understood and poorly characterised. ${ }^{12}$ Whether greater biological specificity of interferon $\gamma$ (IFN $\gamma$ ) release assays (IGRAs), compared with the traditional tuberculin skin test, is associated with identification of LTBI at a higher prospective risk of $\mathrm{TB}$ remains a subject of considerable debate and the focus of two recent meta-analyses. $^{3} 4$ However, discordance in

\section{Key messages}

What is the key question?

- How well does screening with QuantiFERON tuberculosis (TB) Gold-In tube inform prospective TB risk in recent adult contacts of smear positive pulmonary and other less infectious forms of TB?

What is the bottom line?

- A positive QuantiFERON tuberculosis (TB) Gold-In Tube (QFT) is equally valuable in predicting high risk of developing TB within 2 years for young adult contacts of smear positive pulmonary and other forms of TB. For older contacts ( $\geq 36$ years), the positive predictive value of QFT loses significance.

\section{Why read on?}

- This study reports on the effectiveness of a QuantiFERON TB Gold-In Tube based single-step model of screening for recent adult contacts.

outcomes and conclusions between the meta-analyses is striking and attributable to differences in study selection that significantly influence the context of LTBI study. The analysis of Rangaka $e t a l^{3}$ was confined to studies performed in low and middle income countries with a moderate to high TB burden. In contrast, Diel $e t a l^{4}$ analysed a smaller number of studies from high income, low TB prevalence settings. Even for the two studies performed in a low prevalence setting that have specifically evaluated TB risk with IGRA screening for recent adult TB contacts of smear positive pulmonary (SP) TB, there is a fourfold difference between them in reported prospective 2-year TB risk. $^{5} 6$ Important differences in study design, objectives and population characteristics are likely to have contributed to this difference.

A clear need therefore exists for further studies to better inform IGRA based screening policies. ${ }^{7}$ Since January 2007, we have performed QuantiFERON TB Gold-In Tube (QFT) based single-step adult contact screening at our centre that is adapted from 2006 UK National Institute for 
Health and Clinical Excellence guidance. ${ }^{9}$ All contacts were followed prospectively for occurrence of secondary TB events. Here we present the findings of a longitudinal cohort analysis that quantifies 2-year TB risk in contacts of both SP and other nonsmear positive (NSP) TB. We examine whether prospective TB risk with QFT defined LTBI differs between recent adult contacts according to their type of exposure and age and present indices of effectiveness with a single-step model of screening with QFT in clinical practice.

\section{METHODS}

\section{Study population}

The study population included all notified cases of active TB and associated asymptomatic contacts entering the screening programme between 1 January 2007 and 5 June 2009. Cases of active TB in the contact population were recorded until 15 March 2010.

\section{Active TB}

A diagnosis of active TB was made in accordance with accepted criteria. ${ }^{9}$ IGRA testing is not included in the diagnostic evaluation for active TB in adults at our centre. For cases with culture confirmation, genotyping of the isolate using PCR based identification of variable number of tandem repeats for mycobacterial interspersed repetitive units (VNTR-MIRU) of 15 discriminatory loci ${ }^{10}$ was performed at the HPA Regional Mycobacteriology Laboratory in Birmingham.

\section{Screening algorithm}

We designed and employed a single-step model of screening for adult contacts (age $\geq 16$ years) that was adapted from $2006 \mathrm{UK}$ National Institute for Health and Clinical Excellence guidance ${ }^{9}$ (figure 1). In brief, contact tracing procedures were initiated immediately after index notification and screening for active TB performed using a symptom questionnaire. QFT testing was performed according to the manufacturer's instructions (Cellestis, a QIAGEN company, Hilden, Germany) in all contacts aged below 36 years and in contacts of any age exposed to SP TB after 8-12 weeks.

All QFT positive adult contacts under 36 years were offered chemoprophylaxis with rifampicin and isoniazid for 3 months (3RH), unless drug resistance was identified in the source case. QFT positive contacts aged $\geq 36$ years and younger QFT positive contacts who declined chemoprophylaxis had active follow-up with clinical assessment and a chest radiograph at 6-monthly intervals for 2 years after index notification. QFT negative adults and untested contacts aged $\geq 36$ years were followed-up once after 3 months. Passive follow-up is maintained for all contacts who remained in the region through a centralised system of rapid access and our local networked electronic TB database (TBIT) (online supplement).

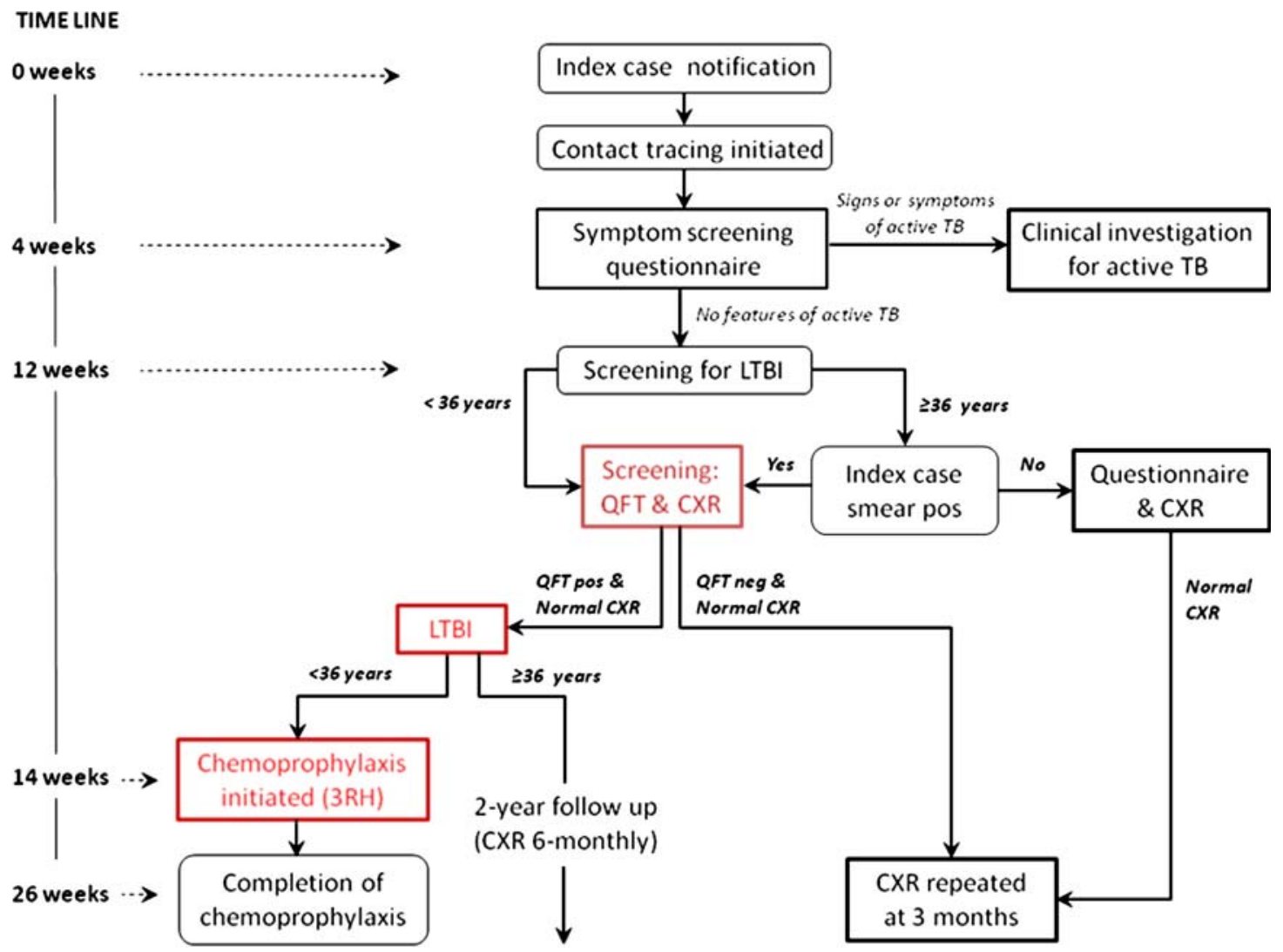

Figure 1 Study algorithm and timeline for single-step adult contact screening using QuantiFERON-tuberculosis (TB) Gold-In Tube (QFT). The algorithm was designed as an adaptation of the UK National Institute for Health and Clinical Excellence 2006 guidelines for a single-step interferon $\gamma$ release assay based protocol. Key similarities with the guideline are delayed QFT testing after index case notification; restriction of QFT testing in persons aged 36 years and above to contacts of smear positive pulmonary disease; and provision of prophylactic chemotherapy for close contacts aged below 36 years with a positive QFT result. Important differences include absence of preliminary screening with the tuberculin skin test for early identification of latent and active TB; absence of any influence of prior BCG vaccination status on the algorithm for screening; and extended follow-up of healthy screened contacts who either decline or do not meet eligibility criteria for prophylactic chemotherapy. The labels of early and late disease refer to whether or not TB was potentially preventable with screening (see Methods section). CXR, chest radiograph; LTBI, latent infection with Mycobacterium tuberculosis. This figure is only reproduced in colour in the online version. 


\section{Screening timeline and categorisation of contact TB}

By definition, contact TB risk was modifiable with screening if an opportunity for chemoprevention existed prior to disease onset (figure 1). On this basis, contact TB identified $\leq 14$ weeks after index notification (early and co-prevalent TB) was not preventable and TB occurring after 14 weeks (late disease) was potentially modifiable with screening (online supplement).

\section{Statistical methodology}

The primary outcome of this study was cumulative 2-year risk of active TB in QFT positive adult contacts of SP and NSP TB, using our screening algorithm. NSP TB included all nonpulmonary cases and smear negative pulmonary TB. Secondary outcomes were quantification of performance with QFT as a single-step screening tool for targeted delivery of chemoprophylaxis; comparison of TB rates between younger ( $<36$ years) and older adults; and characterisation of factors associated with failure of the screening model to prevent contact TB. All contact cases were included for characterisation of factors associated with screening failure. Contacts with protocol deviation (inappropriate QFT testing and/or inappropriate chemoprophylaxis) and QFT tested contacts diagnosed within 2 weeks of testing (QFT result representative of active TB and not LTBI) were excluded from QFT based longitudinal analyses.

Between-group statistical comparisons were performed using the independent $t$ test (parametric variables) and MannWhitney test (non-parametric variables). Kaplan Meier analysis was performed to estimate TB risk in the prespecified contact groups and compared between them using the log-rank test. The number needed to screen (NNS) and number needed to treat (NNT) were calculated (online supplement) as respective indices of screening and therapy effectiveness with our model.

Performance measures of QFT as a screening tool included population independent (sensitivity, specificity and positive and negative likelihood ratios) ${ }^{11}$ and population dependent (positive predictive value and negative predictive value; online supplement) parameters. These were computed with contingency tables for all contacts and separately in contacts of SP and NSP TB. Contingency tables were also used to calculate TB rate ratios. The 95\% CIs were determined using Wald's method and significance testing performed with the $\chi^{2}$ test.

Forward stepwise logistic regression was performed (online supplement) to model the association of prespecified variables (see online supplementary table S2) with the QFT result in all tested contacts.

SPSS V.16 (SPSS, Inc.) was used to perform survival analyses, and significance testing with the log rank test and logistic regression. Prism V.5 (GraphPad Software, Inc., California, USA) was used to perform analyses of contingency tables. For all analyses, the significance threshold was $<0.05$.

The study was approved by the Leicestershire TB Management Board; the local research and ethics committee was consulted and formal ethical approval was not required. No external funding was obtained.

\section{RESULTS}

\section{Study population and uptake to the screening programme}

Close contacts were identified for 505 of $628(80 \%)$ notified TB cases (see online supplementary table S1). Overall, 1769 of 2401 contacts $(74 \%)$ were aged above 15 years and included in the study (figure 2). The overall culture confirmation rate in the cohort with index $\mathrm{TB}$ was $60 \%$ and significantly higher for pulmonary $\mathrm{TB}$, compared with non-pulmonary disease $(76 \%$ vs $41 \%$; $\mathrm{p}<0.001)$.
In 34 adults (1.9\%) protocol deviations were identified, and comprised inappropriate screening and treatment of older adults (figure 2). A total of 817 of 1100 eligible adults (74.4\%) were QFT tested. The mean $( \pm \mathrm{SD})$ time to testing after index notification was $95 \pm 22$ days. The proportion of eligible contacts tested did not differ across prespecified subgroups, stratified by type of disease exposure or age. In all, 98 of 147 (66.0\%) eligible QFT positive contacts accepted chemoprophylaxis and treatment completion confirmed in 76 (74.6\%) of this group. In all treated subjects, chemoprophylaxis was well tolerated with no adverse events requiring cessation of therapy.

\section{Outcome of QuantiFERON testing}

Overall, $215(26.3 \%)$ of the 817 appropriately tested contacts were QFT positive (figure 2). An indeterminate result occurred for one contact. Five QFT positive contacts were diagnosed with TB at the time of screening (ie, within 2 weeks of testing). Modelling identified the contact's age, place of birth (UK or abroad) and disease type of the index case (SP or NSP) to be independently associated with the QFT result. Of these, index disease type was most strongly associated with an OR $(95 \% \mathrm{CI})$ for a positive QFT of 5.6 (3.4-9.1) for contact with SP TB, compared with NSP disease (see online supplementary table S2). Overall, the proportion of contacts who were QFT positive was $20.2 \%$ for non-pulmonary, $18.6 \%$ for smear negative pulmonary and $34.8 \%$ for SP TB, respectively.

\section{Secondary TB in contacts}

Contacts were observed prospectively for a median of 717 days (range 283-1157 days). Based on published migration figures (online supplement), ${ }^{12}$ we estimated 95 contacts would have been lost to follow-up, with a risk of TB occurring in less than two contacts over the median period of observation. In all, 43 cases of active TB were identified in contacts of 37 index cases. A table summarising details for each contact case is provided (see online supplementary table 3). One of 35 tested adults was HIV positive at the time of notification. A positive culture for $M$ tuberculosis was obtained in 26 contact cases. For the remainder, TB was diagnosed on the basis of compatible clinical symptoms, signs and radiological features coupled with either clinical and radiological response following a full course of antituberculous therapy alone $(\mathrm{N}=9)$ or additionally with histological evidence of necrotising granulomatous inflammation $(\mathrm{N}=8)$ (see online supplementary table S2). Genotype data for the isolate were available for 22 pairs of index cases and associated contact cases and genotypically matched in 18 pairs $(82 \%)$. There were no differences in characteristics between subgroups with matched and unmatched results.

The median time to contact TB after notification of the index case was 171 days (IQR 93-287 days). Co-prevalent TB was diagnosed in one contact after 16 days and nine further contacts were categorised with early disease. For the remaining 33 contacts $(76.7 \%)$ with late disease, three primary factors associated with failure to prevent TB were identified (online supplementary figure 1): non-adherence with the screening model $(\mathrm{N}=12)$; ineligibility for chemoprophylaxis due to age $(\mathrm{N}=15)$; and QFT negative at time of screening $(\mathrm{N}=6)$.

\section{Testing with QuantiFERON and TB risk in contacts}

For untreated contacts, the overall 2-year $\mathrm{TB}$ rate was $2.5 \%$ (95\% CI 1.7 to 3.2) (table 1). Contacts of SP TB had a 4.8-fold higher risk of TB than contacts of NSP TB $(p<0.001$; figure 3$)$. There was no difference in the rate of $\mathrm{TB}$ between older and 


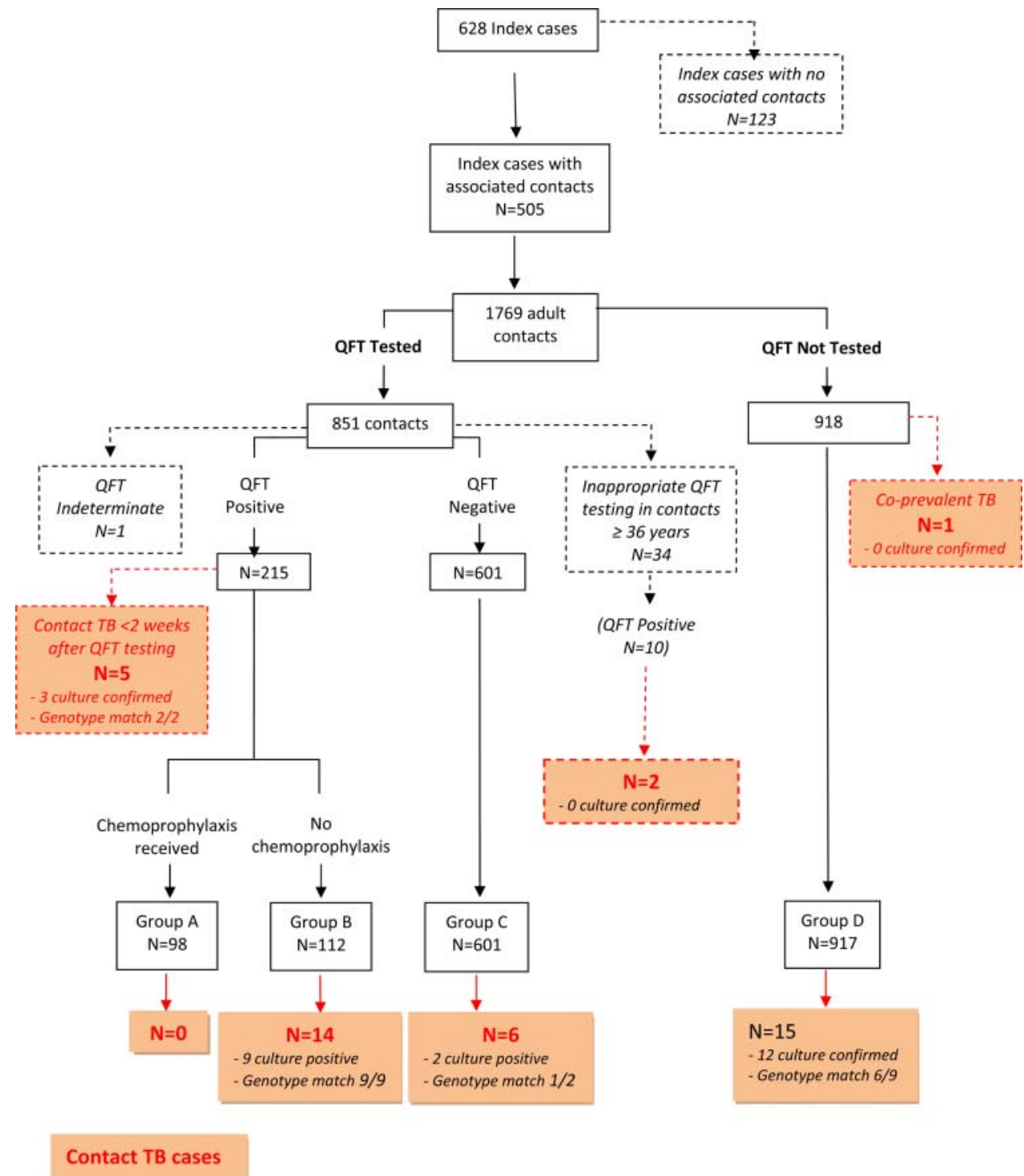

Algorithm followed; group included for longitudinal analysis
Group not included for longitudinal analysis

Figure 2 Stratification of the study cohort and secondary contact tuberculosis (TB) events, according to the screening algorithm. Longitudinal analysis was restricted to contacts who adhered with screening criteria for QuantiFERON TB Gold-In Tube (QFT) testing (see Methods section). Overall, 41 contacts who included eight TB events were excluded from these analyses. Baseline characteristics of included contacts are presented (table 2). Subgroups defined by the screening pathway are summarised. Group A: QFT positive contacts who received chemoprophylaxis. Group B: QFT positive contacts who did not have chemoprophylaxis. This includes smear positive contacts aged over 35 years (ineligible for treatment) and any QFT positive adult contact under 36 years who either declined or did not attend for treatment. Group C: QFT negative contacts who did not receive chemoprophylaxis. This includes tested smear positive contacts aged over 35 years and all tested adult contacts below 36 years. Group D: QFT untested contacts. This includes all contacts of non-smear positive TB, aged over 35 years (ineligible for testing) and adult contacts below 36 years who did not attend for testing. This figure is only reproduced in colour in the online version.

younger contacts (rate ratio $(95 \% \mathrm{CI}))=1.1 \quad(0.6$ to 2.1$)$, $\mathrm{p}=0.678)$.

A total of 41 of 1769 contacts $(2.3 \%)$ were excluded from QFT based analyses of TB risk and screening performance. This subgroup included eight contact TB events of whom one had co-prevalent disease, five were diagnosed at the time of QFT testing and two were cases that occurred in inappropriately screened contacts (figure 2). Summary characteristics of the cohort included are summarised in table 2. For untreated QFT positive contacts, the 2-year TB risk (95\% CI) was $13.4 \%(7.7$ to 21.1) (table 1) and did not differ between contacts of SP and NSP disease (figure 3). There was no difference in the IFN $\gamma$ response of QFT positive contacts who did and did not develop $\mathrm{TB}$, either in the proportion with an IFN $\gamma$ titre $>10 \mathrm{IU} / \mathrm{ml}$ (15.9\% for non-progressors and $15.4 \%$ for progressors to TB; $\mathrm{p}=0.962)$ or in the IFN $\gamma$ titre when this was below $10 \mathrm{IU} / \mathrm{ml}$ (1.1-fold difference, $95 \%$ CI 0.6 to $1.9 ; \mathrm{p}=0.762$ ).

Overall, QFT had a sensitivity of $70 \%$ and specificity of $85.9 \%$ for identifying LTBI progressing to TB at 2 years in our study cohort, using the single-step model. These and other indices of screening performance were comparable between untreated contact subgroups of SP and NSP TB (table 1). A positive QFT result informed a significantly higher 2-year TB risk in all prespecified subgroups except older contacts of SP disease. 
Table 1 Two-year tuberculosis risk in untreated adult contact subgroups and indices of performance with QFT for screening

\begin{tabular}{|c|c|c|c|c|}
\hline & \multirow[b]{2}{*}{ All adult contacts } & \multicolumn{3}{|c|}{ Subgroups of contact exposure } \\
\hline & & Non-smear positive & Smear positive (all) & Smear positive ( $\geq 36$ years) \\
\hline \multicolumn{5}{|c|}{ 2-year risk [cases/cohort size]† } \\
\hline All untreated & 2.5 (1.7 to 3.2$)$ [39/1669] & $1.3(0.8$ to 2.1$)[16 / 1263]$ & 6.2 (4.6 to 8.1$)[23 / 406]$ & 6.5 (3.4 to 10.5$)[12 / 211]$ \\
\hline QFT positive & 13.4 (7.7 to 21.1$)[14 / 112]$ & 13.7 (4.6 to 31.1$)[4 / 32]$ & $13.4(6.8$ to 22.8$)[10 / 80]$ & $8.9(3.3$ to 19.1$)[5 / 62]$ \\
\hline QFT negative & $1.1(0.4$ to 2.3$)[6 / 601]$ & 0.3 (0.01 to 1.7$)[1 / 371]$ & $2.4(0.8$ to 5.4$)[5 / 230]$ & $3.4(0.8$ to 9.4$)[3 / 98]$ \\
\hline Rate ratio (QFT pos: All) $\ddagger$ & 4.9 (2.7 to 8.7$) p<0.001$ & 8.9 (3.1 to 25$) p<0.001$ & $2.1(1.02$ to 4.2$) p=0.041$ & $1.4^{*}(0.5$ to 3.9$) p=0.503$ \\
\hline Prevalence QFT positive $/ \%$ & $26.3(23.2$ to 29.0$)$ & $19.5(16.0$ to 23.0$)$ & 34.5 (29.6 to 39.4$)$ & 39.1 (31.5 to 46.7$)$ \\
\hline Sensitivity§ & 70.0 (45.7 to 88.1 ) & 80.0 (28.4 to 99.5$)$ & 66.7 (38.4 to 88.2$)$ & 62.5 (30.6 to 86.3$)$ \\
\hline Specificity§ & 85.9 (83.0 to 88.4$)$ & 93.0 (89.9 to 95.3$)$ & $76.3(71.0$ to 81.0$)$ & 62.5 (54.6 to 69.8$)$ \\
\hline PPV§ & $12.5(7.0$ to 20.1$)$ & 12.5 (3.5 to 29.0$)$ & $12.5(6.2$ to 21.8$)$ & 8.1 (2.7 to 17.8$)$ \\
\hline NPV§ & 99 (97.8 to 99.6$)$ & 99.7 (98.5 to 99.9$)$ & $97.8(95.0$ to 99.3$)$ & 96.9 (91.3 to 99.4$)$ \\
\hline $\mathrm{LR}^{+}$ & $4.95(3.5$ to 7.0$)$ & $11.4(6.5$ to 20.0$)$ & $2 \cdot 8(1.9$ to 4.2$)$ & $1.7^{\star}(0.94$ to 3.0$)$ \\
\hline $\mathrm{LR}^{-}$ & 0.35 (0.18 to 0.68$)$ & $0.22 *(0.04$ to 1.2$)$ & $0.44(0.21$ to 0.90$)$ & $0.6^{*}(0.24$ to 1.5$)$ \\
\hline Number needed to screen & $35.0(21.5$ to 93.1$)$ & 41.5 (20.0 to 432$)$ & $40.0^{*}(-18.3$ to 217$)$ & $105^{*}(-39.6$ to 22.5$)$ \\
\hline Number needed to treat & 6.8 (3.8 to 9.9 ) & 6.5 (3.6 to 33.8 ) & $7.0(4.5$ to 15.0$)$ & Not applicable \\
\hline \multicolumn{5}{|c|}{ 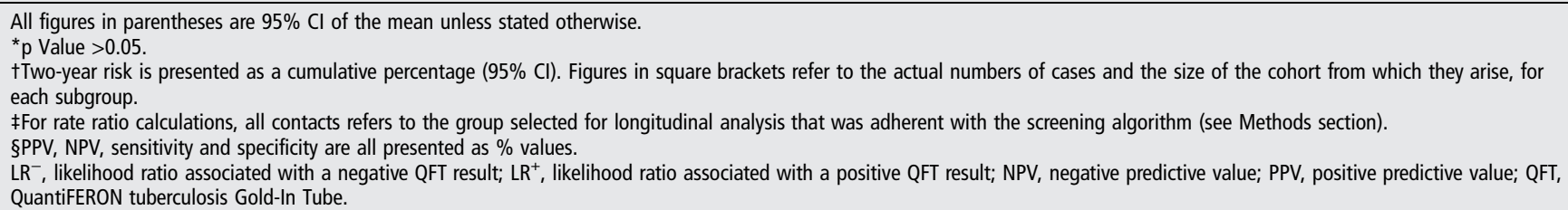 } \\
\hline
\end{tabular}

For this population, there was no difference between the overall disease rate and the rate in the QFT positive subgroup (rate ratio $=1.4(95 \%$ CI 0.5 to 3.9$), \mathrm{p}=0.503$; table 1$)$. Based on the prevalence of QFT defined LTBI in our population, we estimated a NNS of 35 (95\% CI 22 to 93) contacts with our model to identify one adult developing TB over 2 years, without chemopreventive therapy (table 1 ).

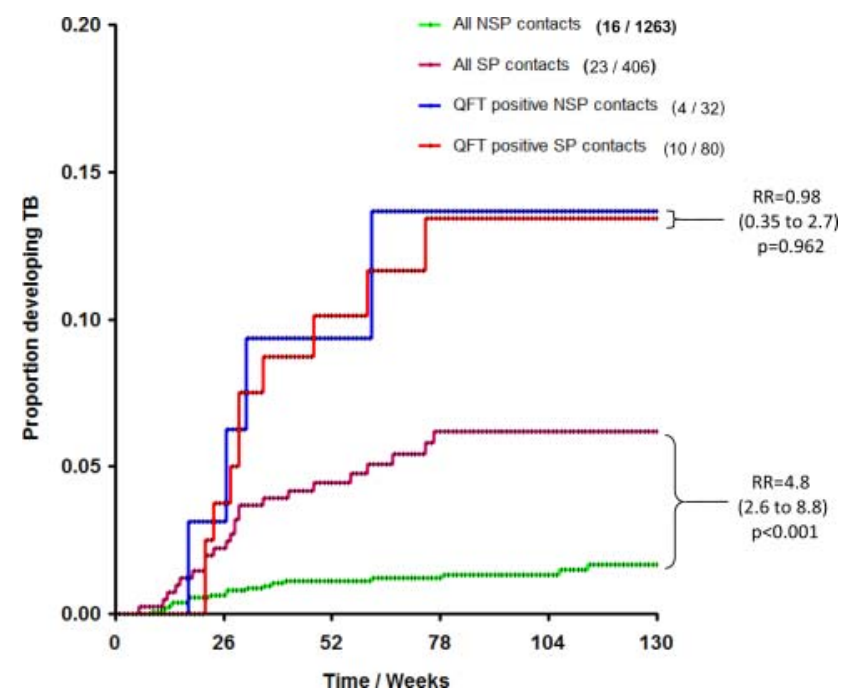

Figure 3 Overview of prospective 2-year tuberculosis (TB) risk in untreated adult contacts, stratified by exposure to smear positive pulmonary (SP) and non-smear positive (NSP) TB. The survival curves for TB occurrence are presented for all contacts of NSP and SP TB and for the subpopulations of each who were QuantiFERON TB Gold-In Tube (QFT) tested and positive. The number of cases and size of the cohort from which they arise are presented with the key: Cases/Size of cohort. RR=2-year rate ratio $(95 \% \mathrm{Cl})$ of disease calculated between exposure subgroups (SP to NSP), as indicated. This figure is only reproduced in colour in the online version.
Six cases arose in contacts who were QFT negative at the time of screening, giving QFT a negative predictive value of 99\% (95\% CI 97.8 to 99.6) (table 1). Three out of four cases retested at the time of presentation with active disease were IGRA positive (two QFT, one T-SPOT.TB). All six cases were HIV seronegative. A positive culture was obtained for two contact cases and strain genotyping identified a match between the index case and contact for one of the two pairs (see online supplementary table S3).

No contacts who received chemoprophylaxis developed TB (figure 2). The NNT of QFT positive contacts with $3 \mathrm{RH}$ to prevent one case of TB over 2 years was 6.8 (95\% CI 3.8 to 9.1), and similar for contact subgroups of SP and NSP disease (table 1).

\section{DISCUSSION}

In this study, we present longitudinal outcomes with a singlestep QFT based programme of screening for adult contacts of TB. We have quantified 2-year TB risk for QFT positive LTBI in contacts of both SP and NSP TB and show that while a fivefold difference exists in the background rate of TB between the two cohorts, there is no difference in the TB rate between subgroups with QFT defined LTBI. We also demonstrate that the positive predictive value of QFT is lower in older contacts of SP TB and may not usefully risk stratify this group for targeted chemoprophylaxis.

Our 2-year case rate of $13.4 \%$ was comparable with the rate reported by Diel et $a l^{5}$ and considerably higher than the figure of $3.1 \%$ reported by Kik et al. ${ }^{6}$ However, the latter included only immigrant contacts and the low positive predictive value of IGRAs supported their view that these assays discriminate poorly between recently acquired LTBI (at higher risk of secondary $\mathrm{TB}$ ) and remote infection. ${ }^{13}$ In our study, older contacts were more likely to have remotely acquired LTBI, as evidenced by a greater proportion who were foreign born (table 2) and an independent association of increasing age with a positive QFT 
Table 2 Characteristics of adult contacts and subgroups included for QFT based longitudinal analyses

\begin{tabular}{|c|c|c|c|c|c|c|c|}
\hline & \multirow[b]{3}{*}{$\begin{array}{l}\text { All adult contacts } \\
(\mathrm{N}=1769)\end{array}$} & \multicolumn{6}{|c|}{ Contacts for QFT based longitudinal analysis $(\mathrm{N}=1728)$} \\
\hline & & \multicolumn{3}{|c|}{ Non-smear positive TB contact $(\mathrm{N}=1284)$} & \multicolumn{3}{|c|}{ Smear positive TB contact $(\mathrm{N}=444)$} \\
\hline & & $\begin{array}{l}16-35 \text { years } \\
(\mathrm{N}=646)\end{array}$ & $\begin{array}{l}\geq 36 \text { years } \\
(\mathrm{N}=638)\end{array}$ & $\begin{array}{l}\text { Sig ( } p \\
\text { value) }\end{array}$ & $\begin{array}{l}16-35 \text { years } \\
(\mathrm{N}=233)\end{array}$ & $\begin{array}{l}\geq 36 \text { years } \\
(\mathrm{N}=211)\end{array}$ & $\begin{array}{l}\text { Sig }(p \\
\text { value) }\end{array}$ \\
\hline \multicolumn{8}{|l|}{ Gender } \\
\hline Male (\%) & $884(50.0)$ & $330(51.4)$ & $318(50.2)$ & 0.695 & $117(50.2)$ & $100(46.1)$ & 0.569 \\
\hline \multicolumn{8}{|l|}{ Age group (\%) } \\
\hline $16-35$ years & $885(50.0)$ & & & & & & \\
\hline$\geq 36$ years & $884(50.0)$ & & & & & & \\
\hline \multicolumn{8}{|l|}{ Ethnicity (\%) } \\
\hline South Asian & $1273(72.1)$ & $501(77.8)$ & $489(76.8)$ & $<0.001$ & $130(56.5)$ & $124(59.0)$ & 0.26 \\
\hline Black & $140(8.0)$ & $70(10.9)$ & $38(6.0)$ & & $20(8.7)$ & $9(4.3)$ & \\
\hline White & $259(14.6)$ & $37(5.7)$ & $75(11.8)$ & & $71(30.9)$ & $71(33.8)$ & \\
\hline Other & $90(5.1)$ & $36(5.6)$ & $35(5.5)$ & & $9(3.9)$ & $6(2.9)$ & \\
\hline \multicolumn{8}{|l|}{ Origin $(\%)$} \\
\hline Foreign born (\%) & $518 / 876(59.1)$ & $231 / 409(56.5)$ & 107/125 (85.6) & $<0.001$ & $57 / 165(34.5)$ & $100 / 145(69.0)$ & $<0.001$ \\
\hline \multicolumn{8}{|c|}{ Index case disease type (\%) } \\
\hline Non-smear positive & $1323(74.8)$ & & & & & & \\
\hline $\begin{array}{l}\text { Pulmonary smear } \\
\text { positive }\end{array}$ & $446(25.2)$ & & & & & & \\
\hline $\begin{array}{l}\text { Household } \\
\text { contact }(\%)\end{array}$ & $910(51.4)$ & $367(56.8)$ & $361(56.6)$ & 0.955 & 79 (33.9) & $84(39.8)$ & 0.202 \\
\hline Partner contact (\%) & $285(16.1)$ & $86(13.3)$ & $153(24.0)$ & $<0.001$ & $18(7.7)$ & $21(10.0)$ & 0.502 \\
\hline
\end{tabular}

(see online supplementary table S2). Our finding that a positive QFT result was not significantly discriminatory for this group is therefore consistent with Kik et al and favours the view that QFT is less effective as a screening tool in populations with a significant burden of remote infection. Although limitations of QFT support the practice of age-limited screening, ${ }^{9} 15$ of 43 contact cases $(35 \%)$ in our cohort were potentially preventable with age-extended delivery of chemoprophylaxis. A clear need exists to refine screening strategies, rethink traditional policies and encourage development of more specific biomarkers to enable safe and selective provision of chemoprophylaxis for this group.

Previous studies have not formally examined the role of QFT for screening contacts of NSP TB. Although limited to younger adults, we found QFT performed better as a screening test for targeting chemoprophylaxis in this group, compared with its use in SP contacts. This is quantified as a higher rate ratio of prospective TB (table 1) and reflects the retained positive predictive value of QFT on a background of lower TB risk for the group. Our data therefore support a role for QFT in screening all young adult contacts.

Six of 601 QFT negative contacts in our cohort developed TB. It is unlikely that immunodeficiency was a significant contributor, as evidenced by HIV seronegativity and a positive IGRA at the time of presentation with active disease. Although early testing prior to establishment of adaptive immunity may yield false negative IGRA results, the time to testing in our QFT negative contacts developing TB was 8-12 weeks after index notification (see online supplementary table S3) and did not differ with the interval in QFT positive contact cases. It is possible that disease represented new infection, acquired after screening. In keeping with this, we found mismatch in strain genotype between the index and contact for one of the two culture positive cases and for four of 22 cases overall. Although we used 15 loci MIRU-VNTR for strain typing it is unlikely that the current standard of 24 loci would have affected our outcomes significantly. ${ }^{14}$ Interestingly, the sensitivity we report for QFT as a biomarker of TB risk is very similar to the pooled sensitivity of the assay for detecting active TB in meta-analyses, ${ }^{15} 16$ suggesting that clinically relevant $M$ tuberculosis infection may not be identified with QFT in a small number of individuals. Nevertheless, the negative predictive value of the test in a high risk setting of recent $\mathrm{TB}$ contact is excellent and a reliable biomarker for safely withholding chemoprevention in adults (table 1).

We determined a 14-week interval after index notification would be needed with our model before chemopreventive therapy could be started to modify TB risk. On this basis, 10 contact cases $(23.2 \%)$ with early disease were unpreventable. This proportion is comparable with a recent study that reported $35 \%$ of contact cases occurring within 3 months of index notification. ${ }^{17}$ Although accelerated screening to capture early progressors may increase the likelihood of false negative IGRAs, ${ }^{18-21}$ a two-phase screening strategy with immediate testing after index notification and provision of early chemoprophylaxis to QFT positive contacts, followed by a second phase of testing after 6-12 weeks in QFT negative contacts, may be effective. As immediate screening for active TB in our model was performed with a symptom questionnaire alone, it is possible that a proportion of early cases represented asymptomatic co-prevalent disease that would be unpreventable with any screening.

Our study has some limitations. As a cohort study, investigators were not blinded and some deviations from the screening protocol occurred. However, the number of such deviations was sufficiently small to be excluded without introducing significant bias. As culture confirmation was not achieved for a proportion of associated index and contact cases, we are unable to characterise transmission patterns with certainty and the performance of QFT for the subset of true progressors. However, our 
primary objective was to present the use of single-step QFT screening for implementation in a cohort that is typical of the UK and other low prevalence countries with a high proportion of immigrant TB. In this respect, effectiveness of our model, expressed as the NNS and NNT to prevent one case of TB, compares favourably with screening using the tuberculin skin test, for which the estimated NNS (63-167) and NNT (7-177) are considerably higher. ${ }^{22}$ In conclusion, targeted delivery of 3RH chemoprophylaxis using a QFT based single-step screening strategy is effective and resource efficient for implementation in recent close contacts of active TB below the age of 36 years. Provision of chemoprophylaxis in older contacts of SP TB requires consideration, although effective $\mathrm{TB}$ risk stratification may not be achieved with QFT alone for this group.

Acknowledgements The authors acknowledge the special contributions of the Leicester TB nursing service and administrative staff, including Lynda Hewitt and Joanne Lee to data collection and study completion. The authors thank Dr Ruth Green for her advice with data analysis and statistical aspects of the study.

Contributors All listed authors meet the criteria for authorship set forth by the International Committee for Medical Journal Editors. PH and GW designed the study, extracted the clinical data and performed the analysis. PM, EWH, MW, JE and GW conceived the screening model and advised on analysis. MB provided microbiological data and wrote the paper with PH and GW. HT leads the contact screening service provided by the nursing team, NP and HP were involved with IGRA testing. All authors contributed to editing of the final manuscript.

Funding None.

Competing interests None.

Ethics approval The study was discussed with the Leicestershire, Northants and Rutland Research Ethics Committee who advised that formal ethical approval was not required.

Provenance and peer review Not commissioned; externally peer reviewed.

Data sharing statement The authors are fully committed to the principles of data sharing.

\section{REFERENCES}

1 Barry CE III, Boshoff HI, Dartois V, et al. The spectrum of latent tuberculosis: rethinking the biology and intervention strategies. Nat Rev Microbiol 2009:7:845-55.

2 Mack U, Migliori GB, Sester M, et al. LTBI: latent tuberculosis infection or lasting immune responses to M. tuberculosis? A TBNET consensus statement. Eur Respir J 2009:33:956-73.

3 Rangaka MX, Wilkinson KA, Glynn JR, et al. Predictive value of interferon-gamma release assays for incident active tuberculosis: a systematic review and meta-analysis. Lancet Infect Dis 2012;12:45-55.
4 Diel R, Loddenkemper R, Nienhaus A. Predictive value of interferon-gamma release assays and tuberculin skin testing for predicting progression from latent TB infection to disease state: a meta-analysis. Chest 2012;142:63-75.

5 Diel R, Loddenkemper R, Niemann $S$, et al. Negative and positive predictive value of a whole-Blood IGRA for developing active TB-an update. Am J Respir Crit Care Med 2011;183:88-95.

6 Kik SV, Franken WP, Mensen M, et al. Predictive value for progression to tuberculosis by IGRA and TST in immigrant contacts. Eur Respir J 2010;35:1346-53.

7 Mazurek M, Jereb J, Vernon A, et al. Updated guidelines for using interferon gamma release assays to detect mycobacterium tuberculosis infection-United States, 2010. MMWR Recomm Rep 2010:59:1-25.

8 National Institute for Health and Clinical Excellence. Tuberculosis: clinical diagnosis and management of tuberculosis, and measures for its prevention and control. http://www.nice.org.uk. 1-3-2011. Ref Type: Electronic Citation.

9 National Collaborating Centre for Chronic Conditions. Clinical diagnosis and mangement of tuberculosis and measures for its prevention. http://www.rcplondon. ac.uk/pubs/books/TB/index.asp. 2006. Royal College of Physicians. Ref Type: Electronic Citation.

10 Supply P, Allix C, Lesjean S, et al. Proposal for standardization of optimized mycobacterial interspersed repetitive unit-variable-number tandem repeat typing of Mycobacterium tuberculosis. J Clin Microbiol 2006;44:4498-510.

11 Chang KC, Leung CC. Systematic review of interferon-gamma release assays in tuberculosis: focus on likelihood ratios. Thorax 2010;65:271-6.

12 Office for National Statistics. http://www.ons.gov.uk. 2008. Ref Type: Electronic Citation.

13 Kik SV, Franken WP, Arend SM, et al. Interferon-gamma release assays in immigrant contacts and effect of remote exposure to Mycobacterium tuberculosis. Int J Tuberc Lung Dis 2009;13:820-8.

14 Allix-Beguec C, Fauville-Dufaux M, Supply P. Three-year population-based evaluation of standardized mycobacterial interspersed repetitive-unit-variable-number tandem-repeat typing of Mycobacterium tuberculosis. J Clin Microbiol 2008;46:1398-406.

15 Pai M, Zwerling A, Menzies D. Systematic review: T-cell-based assays for the diagnosis of latent tuberculosis infection: an update. Ann Intern Med 2008;149:177-84.

16 Diel R, Loddenkemper R, Nienhaus A. Evidence-based comparison of commercial interferon-gamma release assays for detecting active TB: a metaanalysis. Chest 2010;137:952-68.

17 Lee MS, Leung CC, Kam KM, et al. Early and late tuberculosis risks among close contacts in Hong Kong. Int J Tuberc Lung Dis 2008;12:281-7.

18 Erkens CG, Kamphorst M, Abubakar I, et al. Tuberculosis contact investigation in low prevalence countries: a European consensus. Eur Respir J 2010;36:925-49.

19 Guidelines for the investigation of contacts of persons with infectious tuberculosis. Recommendations from the National Tuberculosis Controllers Association and CDC. MMWR Recomm Rep 2005;54:1-47.

20 Lee SW, Oh DK, Lee SH, et al. Time interval to conversion of interferon-gamma release assay after exposure to tuberculosis. Eur Respir J 2011;37:1447-52.

21 Updated recommendations on interferon gamma release assays for latent tuberculosis infection. An Advisory Committee StatementACS). Can Commun Dis Rep 2008;34(ACS-6):1-13.

22 Rose DN. Benefits of screening for latent Mycobacterium tuberculosis infection. Arch Intern Med 2000;160:1513-21. 